\title{
DETERMINING AN EXTRAORDINARY CONSTANT: A PLS PROJECT
}

\author{
BARBARA BALOSSI ${ }^{1}$, MATTEO BOZZI',3, MAURIZIO ZANI ${ }^{2}$ \\ ${ }^{1}$ Liceo Scientifico e Musicale "G.B.Grassi" , Lecco (ITALY) \\ ${ }^{2}$ Department of Physics, Politecnico di Milano (ITALY) \\ ${ }^{3}$ Liceo Scientifico Statale "Vittorio Veneto", Milano (ITALY)
}

\begin{abstract}
The Italian government recently reformed the examination which concludes the Secondary School study programme. With reference to the Liceo Scientifico, i.e. a specific kind of High School with students specializing in scientific subjects, this reform emphasized the crucial role played by Physics in this curriculum. Consequently, revamping the teaching of Physics should be a primary goal. This change ought to increase the time and energies allotted to experimental didactic activities as well as the use of ad hoc educational videos on Physics phenomena.

Our research team developed an instructional experience based on the experimental measurement of Planck constant $h$, a fundamental Physics constant, run directly by a group of 17 -year old students attending the Liceo Scientifico Statale "G. B. Grassi" located in Lecco, in the north-west of Italy. Moreover, a didactic video of the same experiment was produced by the Experimental teaching lab. ST2 of the Politecnico di Milano in order to foster students' reflection on the same experience as a follow-up activity.This synergistic use of the laboratory activity and the related video can be regarded as a significant novelty in teaching innovation. Finally, in an Open Educational Resources philosophy (OER), this video will be made available on the web site of the Lab. ST2 YouTube channel.

Our project was developed in the context of the Piano Nazionale Lauree Scientifiche, a government programme which funds didactic innovation in Science, Technology, Engineering and Mathematics (STEM) in Higher Education and at Secondary School level.
\end{abstract}

Keywords: Experimental video, Physics, Open Educational Resources

\section{INTRODUCTION}

In the last decade, Italy launched a challenge in the field of scientific culture, promoting activities that have resulted in innovative proposals and models both in teaching and in dissemination. The primary objective is to overcome the preconceptions and prejudices that lead to the differentiation of the knowledge and perception of scientific disciplines between female and male students with respect to STEM subjects and the Piano Nazionale Lauree Scientifiche (PLS) [1].

Educational institutions facilitated a constructive dialogue between Upper Secondary Schools and Universities [2], which work in synergy to find strategies, support and teaching materials to be adopted to overcome the challenge. It starts with Physics: the Scientific High School's course of study was introduced as a characterizing subject in all its options in November 2014 and today is the subject of the second written exam of the State Examination's second written exam which concludes high school.

The aim is for students to acquire the following skills by the end of the fifth class [3]:

1. Observing and identifying phenomena

2. Addressing and solving simple physics problems using the appropriate mathematical tools for their educational path

3. Being aware of the experimental method's various aspects, where the experiment is intended as a reasoned query of natural phenomena, a critical analysis of the data and of the reliability of a measurement process

4. Understanding and evaluating the scientific and technological choices affecting the society they live in

It is necessary to enter into the concrete practice of teaching [4], with the cultural, material and human conditionings that are specific to it. In addition, the chapters of Modern Physics, i.e. Quantum Mechanics and Relativity, occupy 55\% of the year-end Physics curriculum. Arousing interest in these 
topics, with all the baggage of paradoxes and applications, can be quite arduous if approached in a "bookish" way, and worse if they are relegated to the final part of the annual didactic programming, close to the State Examination [5].

The fundamental concepts of the Physics of the microscopic world can captivate the intellectual curiosity of young people, but being far from everyday experience, can also be relegated to an almost mythological collective imagination.

The development of the contemporary world contributes to this status, because it is often interpreted in purely technological terms, with the risk of inhibiting Millennials' intellectual expansion capacity. Why do students at the end of a scientific course address higher studies in non-related disciplines? Is the approach to modern physics so devastating that it leads to a reduction in enrollment in scientific degree courses? We want to demonstrate the exact opposite! Twentieth-century themes can promote learning: through today's technology and starting from a great idea, in a cascade, we pass from observation to the definition of physical law.

The three key points are:

a) Student involvement

b) Investigation and in-depth analysis

c) Constructive action [6]

Research, deepen, present and question previous knowledge: this is what video and graphic apps are for. They allow the visualization of the searched result, because the experiment in itself is usable at any moment during the day and the resolution of what is proposed can be shared and debated among more subjects. The figure of the teacher is at the student's service because, by putting questions on Physics concerning the measurement of the Planck constant, the teacher manages to intrigue the students. This is not only because it has a value that is little present in everyday reality, but because it has allowed the formalization of the concept of energy quantization. The teacher must be the expert in the solution process. [6]

To sum up, we will try to focus on one of the experiments that allowed us to reveal a totally new universe which, until the beginning of the last century, was clouded by prejudices.

\section{METHODOLOGY}

When relations are established between variables and formalized in a mathematical equation, the socalled "constants" appear. Their physical meaning is not always analyzed with sufficient attention and thus associated learning difficulties are generated. In many cases, the analysis provided by textbooks and other information available to students is limited to statements such as "where k is a constant", without adding anything else, or adding, at most, a few sentences of the type "... is a constant that depends on the medium" or "from the choice of the reference system". This information does not sufficiently clarify the nature and physical meaning of the relationship in question. As a result of the absence of a reflection on the field of validity imposed by the "constancy of constants", students often attribute an "absolute" character to them. Consequently, perplexities arise which students do not understand, precisely because of the constants that fully belong to the microscopic world. It is unusual for textbooks to meticulously explain when and why a model is modified and, therefore, the hypotheses on which certain functional relationships are based.

At face values, conventional didactics do not favour discrimination between the different categories of physical constants. It seems that students are not clear on the fact that there are constants of many different types in Physics, nor is it clear what the difference is. The roles played in the knowledge of the physics of the "Planck constant" and the "constant of a spring" are, for example, undoubtedly different and students recognize this, at least in analyzing the order of magnitude. However, the nature of the difference seems to escape their understanding. Universal constants, system parameters, boundary conditions ... all are constant: yet for many students all are confusedly mixed. With alarming frequency, they uncritically read the relationships between the quantities, without understanding their functional meaning [7]. It seems that students lose sight of the conceptual framework associated with the mathematical formula: there remains only a symbolism devoid of the meaning of giving conceptual rigor to its practical application. And without conceptual rigor, quantitative rigor lacks meaning.

These observations emerged, with greater emphasis, precisely in the fifth grade of the scientific high school during the normal course of activities of the last 2 years. Systematically, one finds oneself in 
front of students who do not attribute value to the important role played by Physics in the universal constants: those that appear in the great axioms, the starting points of the theories. For this reason, it is important for them to measure the Planck constant, within a reasonable limit of uncertainty $(10 \%)$, according to the classic Millikan experiment on the photoelectric effect, exploiting the interaction of light with matter. Students physically touch and "see", interpreting the data collected, that the energy transferred to the conduction electrons in a metal (potassium in the case under examination) depends on the frequency $v$ of the incident light and not on the intensity which only determines the number of electrons released.

The law $K_{\max }=h v-W_{k}$ allows the determination of the Planck constant $h$, exposing a photocell to a monochromatic light, having a specific frequency $v$ and wavelength $\lambda$, measuring the kinetic energy $\mathrm{K}_{\max }$ and the $\mathrm{W}_{\mathrm{k}}$ extraction work, proper to the metal considered. This experimental set-up allows the student to test several fronts and the teacher to verify the competences of the learner according to what has been said previously.

In fact it is possible to determine:

a) The characteristic curve of a photocell (graph current - voltage)

b) The kinetic energy of electrons as a function of light frequency

c) The Planck constant

In each phase the student must implement the skills indicated in points $1 ., 3$. and 4 . of the previous Introduction chapter, without forgetting a correct graphical representation of the curves indicated and the calculation of the error. The student must immediately observe (see the competences in points 1. and 2.) that the energy of an electron reaching the anode is defined by: $E=h v-W_{k}-e V$. We find the voltage $V$ to be applied to reduce the current I to 0 (also called $V_{s}=$ counter voltage). Measuring $V_{s}$ for different frequencies, we get the graph $\left(V_{s} ; v\right)$. It is represented by a straight line whose slope is h/e: the value of the constant $h$ is verified experimentally by multiplying by the electronic charge $e$ [8].

But how can this experience be made to be engaging? Bear in mind that we must not overlook students' effectiveness and curiosity. For this reason, we tried to experiment with teaching to learn, problem-based learning, real-time feed-back and cooperative learning through the use of certain videos. Significant moments of experience were chosen to understand the photoelectric effect, focusing on the concepts of modern physics, such as monochromatic electromagnetic radiation, radiation and matter, stopping potential and characteristic curve of a photocell. In particular, students were asked to investigate the topic of black-body radiation in groups, with resources selected by the teacher on the reference text or on the web, to then explain it to classmates also with the support of texts or other teaching materials created for the occasion. Subsequently, the involvement of students and the effectiveness of their lessons increased, starting from the videos of the tangible experience. To keep attention high and immediately verify the teaching's effectiveness, questions were asked in class during the explanation, and the values obtained with tools that aggregate the results in real-time were analyzed: in particular, the graph for the Current - Intensity curve was created according to the stopping potential.

Here is an example [9]:

Beginning of observation

1. Before you begin, what will happen to the metal surface when light strikes it

2. Do you think all intensities of light will liberate electrons? Explain

3. Do you think all wavelengths of light will liberate electrons? Explain

Intro to Photoelectric Effect

1. What can you change in the experiment?

2. Develop a procedure for determining the characteristics of the light necessary to liberate electrons from the metal. Write your procedure and your conclusions below

3. Based on your chosen metal, answer the following questions: a) At which wavelengths was it first possible to remove electrons? b) Which wavelengths were most effective? c) What does intensity change? 
Finally the teacher interacts with the students, in the perspective of the peer review, making the students responsible for a goal to be achieved together in order to enhance the attitudes and skills of each.

\section{RESULTS}

The literature provides several ways to evaluate measured data and determine the exact counter voltage at which the photocurrent starts. The easiest approach is to record the current - voltage characteristic of a photocell by a graph, where the horizontal axis represents the voltage $V$ and the vertical axis the photocathode current I. It is more important for the student to realize that there is an adjustable voltage $\mathrm{V}$ applied between the anode and cathode, usually with the anode at a more negative potential than the cathode. Some of the ejected photoelectrons travel to the anode, and if the electron's kinetic energy is larger than the energy loss while "climbing up" the potential eV, they reach the anode and are registered as a photoelectric current $\mathrm{I}$. $\mathrm{V}_{\mathrm{s}}$ is called the counter voltage, that is the voltage at which the photoelectric current reaches zero. In this experiment the current - voltage traces are recorded for various wavelengths $\lambda$, so the frequency of the incident light is $v=c / \lambda$, where $c$ is the speed of light in a vacuum.

When the frequency of an incident light increases by $\Delta v$, electron energy increases by $h \Delta v$. The counter voltage will then grow by $\Delta V_{s}$ to compensate for the rise in energy. This increase is independent of the work function as long as we use the photocathode's same area. During data collection, the student realizes that the energy of an electron reaching the anode is

$$
\mathrm{E}=\mathrm{h} v-\mathrm{W}-\mathrm{eV}
$$

where $e$ is the elementary charge and $W$ is not just the work function $W_{k}$ of the cathode, as the contact potential between the cathode and the anode is also included in the energy balance. The photocurrent starts to rise at different counter voltages depending on the incident light's wavelength. For our highpressure mercury lamp, this happens at -2 volt with the ultraviolet line, at -0.6 volt with the yellow light and the other wavelength in between. When we plot the counter voltage $V_{s}$ as a function of $v$, we obtain a straight line with the slope

$$
\frac{\Delta V s}{\Delta v}=\frac{\mathrm{h}}{\mathrm{e}}
$$

For a known elementary charge e, we have Planck constant $h$.

The way to calculate the counter voltage for one wavelength into a current - voltage graph is very simple: draw a line through all the measurement points on the left side before the photocurrent starts, then draw a parallel line a few picoampere apart and take the crossing of the photocurrent with this line. Another approach is to take a best fit line through all the points on the left, and another best fit line through all the data points at high photocurrents and then look for the intersection of both lines. A third, purely mathematical approach fits an exponential function to the measured values. A function similar to Shockley Diode equation is used

$$
\mathrm{I}(\mathrm{V})=\mathrm{A} \cdot \mathrm{e}^{\mathrm{B} \cdot(\mathrm{V}-\mathrm{C})-1}
$$

where $A$ is the dark current, B somewhat related to light intensity times photocathode response and C is the counter voltage. The best curve that approximates the data obtained, can be determined by using a spreadsheet and searching among the implemented functions which better represent the set of data obtained. At the end, all the different evaluations give quite similar results for the Planck constant, as it is only calculated from the slope of the threshold voltage versus photon energy [9].

For simplicity, we have chosen to use the first method described, paying attention to the point where the photocurrent starts to barely rise above the baseline on the left side of the diagram from the other currents. This gives us the values of the counter voltage versus frequency of light

Table 1. Counter voltage vs wavelength and frequency of light.

\begin{tabular}{l|l|l|l}
\hline \hline & $\lambda(\mathrm{nm})$ & $v(\mathrm{THz})$ & $\mathrm{V}_{\mathrm{s}}(\mathrm{V})$ \\
\hline \hline
\end{tabular}




\begin{tabular}{c|c|c|c}
\hline \hline 1 & 578 & 519 & 0.95 \\
\hline 2 & 546 & 549 & 1.20 \\
\hline 3 & 436 & 688 & 1.50 \\
\hline 4 & 405 & 741 & 1.80 \\
\hline 5 & 365 & 822 & 2.30 \\
\hline \hline
\end{tabular}

Using a spreadsheet to plot the data in a diagram, we see that the plotted measurement points lie on a straight line with close approximation. A line fitted to the measurement points has a slope of

$$
\frac{\Delta V s}{\Delta v}=\frac{h}{\mathrm{e}}=0.00398 \frac{\mathrm{V}}{\mathrm{THz}}=3.98 \cdot 10^{15} \mathrm{Vs}
$$

This value is experimentally calculated, using the statistical mathematical functions that are present in a normal spreadsheet. Once the collected data has been entered, it is possible to determine, with the Ordinary Least Squares, the value of the slope of the straight line which better fits the data itself. The extraordinary constant is now determined, multiplying with the electronic charge e: $\mathrm{h}=6.37 \cdot 10^{-34} \mathrm{Js}$. Considering that the literature value is $\mathrm{h}=6.62 \cdot 10^{-34} \mathrm{Js}$ we can say that the uncertainty is within the reasonable limit of about $12 \%$ [10].

\section{CONCLUSIONS}

The experimental verification of the Planck constant's value highlighted the different types of approaches to the study of physics. The IMAGINATIVE - CREATIVE approach was a first, enabling students to demonstrate an interest in the images by analyzing the video frames in the experiment's individual phases. The student identifies the fundamental quantities involved and aims, given the specific nature of the course of study, to formulate a report and to prepare graphic representations from them. The production of a video gives tangibility to thought. In fact, this keeps track of the reflections that the students shared with each other and with the teacher, mainly centered on the questioning of previous knowledge. Thus, we can dispel the misconceptions which lie dormant until the end of the high school journey [11]. At this point, the EXPERIMENTAL - APPLICATION approach leads to a reflection on the instrumentation used. The technology evolves at a fast pace and some devices also have applications in everyday life. Therefore, questions are asked about the scientific knowledge that underlies their functioning. The natural conclusion of this type of learning is the CRITICAL - REFLECTIVE approach which, for students of a scientific high school, is always accompanied by a mathematical formalism. This allows a profound analysis of the issue addressed, which inevitably leads to reflections of a historical nature and the epistemology of science. The student is encouraged to deepen the evolution of concepts or the cultural context in which they developed [12].

\section{ACKNOWLEDGEMENTS}

Our project was developed in the context of the Piano Nazionale Lauree Scientifiche, a government programme which funds didactic innovation in Science, Technology, Engineering and Mathematics (STEM) in Higher Education and at Secondary School level.

\section{REFERENCES}

[1] S. Bertelli et al., "Esperienze di didattica della Fisica in diversi livelli del sistema educativo", Annali Online Della Didattica e Della Formazione Docente, vol. 9, no 14 (2017), pp. 2-4, 2018.

[2] M. Zani, M. Bozzi, "La fisica tra la scuola secondaria e l'università. Riflessioni e orientamenti", Nuova Secondaria, vol. XXXVI, no. 1, pp. 84-88, 2018. 
[3] Indicazioni Nazionali Indirizzo Liceo Scientifico e Liceo Scientifico - opzione scienze applicate. Retrieved from

http://www.indire.it/lucabas//kmw_file/licei2010/indicazioni_nuovo_impaginato/_Liceo\%20scienti fico.pdf

[4] G. C. Ghirardi et al., "Introduzione delle idee della Fisica Quantistica ed il ruolo del Principio di Sovrapposizione lineare", La Fisica nella Scuola, vol. XXX, no. 3 Supp. Q7, pp. 46-57, 1997.

[5] L. Martina, "Alcune esperienze introduttive alla Meccanica Quantistica", Retrieved from http://www.dmf.unisalento.it/ martina/allow_listing/scuole/EspMeccQuant.pdf

[6] P. Migliavacca, "Challenge Based Learning", 2019. Retrieved from https://www.youtube.com/watch?v=mVUXv_wuy_A

[7] L. Colombo de Cudmani et al., "Tipi diversi di costanti in fisica e apprendimento significativo della disciplina", La Fisica nella Scuola, vol. XXXI, no. 1, pp. 36, 1998.

[8] Paul A. Tipler, Corso di Fisica. Volume 3. Bologna: Zanichelli, pp 1343 - 1347.

[9] PhET, "L'Effetto Fotoelettrico". Retrieved from https://phet.colorado.edu/it/simulation/photoelectric, 2018

[10] "Determining Planck's constant: Counter voltage method" in LD Physics Leaflets P6.1.4.4, Huerth/Germany: LD Didactic GmbH, 2001.

[11] M. Bozzi, P. Ghislandi, M. Zani et al., "Highlight misconceptions in Physics: a T.I.M.E. project", INTED2019 (XIII International Technology, Educational and Development Conference) Valencia (Spain) 11-13/3/2019 - Proceedings of INTED2019 pp. 2520-2525, 2019.

[12] L. Ludovico, "Processi di appropriazione nello studio della fisica quantistica: analisi di una sperimentazione didattica in una quinta liceo scientifico", pp. 85-87, Bologna: Università di Bologna, 2016. Retrieved from http://amslaurea.unibo.it/view/cds/CDS8025/ 